\title{
Optimal inspiratory pressure for face mask ventilation in paralyzed and unparalyzed children to prevent gastric insufflation: a prospective, randomized, non-blinded study Pression inspiratoire optimale pour ventilation au masque facial évitant l'insufflation gastrique chez des enfants paralysés et non paralysés: une étude randomisée sans insu
}

\author{
Ji-Hyun Lee, MD • Haesun Jung, MD • Eun-Hee Kim, MD • In-Kyung Song, MD • \\ Hee-Soo Kim, MD, PhD • Jin-Tae Kim, MD, PhD \\ Received: 31 August 2017/Revised: 25 April 2018/Accepted: 17 May 2018/Published online: 11 July 2018 \\ (C) Canadian Anesthesiologists' Society 2018
}

\begin{abstract}
Background Gastric insufflation is common during face mask ventilation and results in unfavourable respiratory events in children. The purpose of this study was to evaluate the effect of a muscle relaxant on gastric insufflation and determine the optimal inspiratory pressure during face mask ventilation in children.

Methods Children aged one month to five years were randomly assigned to neuromuscular blocker (NM) or nonneuromuscular blocker (non-NM) groups. After administering intravenous anesthetics, face mask ventilation commenced via pressure-controlled mechanical ventilator. Initial inspiratory pressure was 10 $\mathrm{cmH}_{2} \mathrm{O}$ and was increased by $2 \mathrm{cmH}_{2} \mathrm{O}$ until gastric insufflation was detected via gastric ultrasonography or epigastric auscultation. The primary outcome was the difference in the inspiratory pressure that causes gastric insufflation between the two groups. Diagnostic methods that detect gastric insufflation first were also evaluated.

Results There was no significant difference in the median [interquartile range] inspiratory pressure inducing gastric
\end{abstract}

J.-H. Lee, MD · H. Jung, MD · E.-H. Kim, MD

H.-S. Kim, MD, PhD - J.-T. Kim, MD, PhD ( $\varangle)$

Department of Anesthesiology and Pain Medicine, Seoul

National University Hospital, Seoul National University College

of Medicine, \# 101 Daehakno, Jongnogu, Seoul 03080, Republic

of Korea

e-mail: jintae73@gmail.com

I.-K. Song, MD

Department of Anesthesiology and Pain Medicine, Asan Medical Center, University of Ulsan College of Medicine, Seoul,

Republic of Korea insufflation between the non-NM $(n=52)$ and NM groups $(n=60)\left(18\right.$ [16-18] $\mathrm{cmH}_{2} \mathrm{O}$ vs 18.0 [16-20] $\mathrm{cmH}_{2} \mathrm{O}$; median difference, $\mathrm{O} \quad \mathrm{cmH}_{2} \mathrm{O} ; 95 \%$ confidence interval [CI], 0 to 2; $P=0.57$ ). The incidence of gastric insufflation increased with increasing inspiratory pressure. Gastric insufflation was detected first by ultrasonography in $44 \%$ and by epigastric auscultation in 19\% of the non-NM group (difference in percentage, 25\%; 95\% CI, 6 to 42; P $=0.006)$ and by ultrasonography in $73 \%$ and by epigastric auscultation in $7 \%$ of the NM group (difference in percentage, 66\%; 95\% CI, 50 to 78; $\mathrm{P}<0.001$ ).

Conclusions A neuromuscular blocking agent has minimal effect on the inspiratory pressure that causes gastric insufflation during face mask ventilation in children.

Trial Registration www.clinicaltrials.gov (NCT024715 21); registered 15 June 2015.

\section{Résumé}

Contexte L'insufflation gastrique est fréquente au cours de la ventilation au masque et entraîne des événements respiratoires délétères chez les enfants. L'objectif de cette étude était d'évaluer l'effet d'un relaxant musculaire sur l'insufflation gastrique et de déterminer la pression inspiratoire optimale pendant la ventilation au masque des enfants.

Méthodes Des enfants âgées d'un mois à cinq ans ont été affectés de manière aléatoire dans un groupe recevant un bloqueur neuromusculaire (groupe NM) ou dans un groupe n'en recevant pas (groupe non-NM). La ventilation au masque avec un ventilateur mécanique à contrôle de pression a commencé après l'administration intraveineuse des anesthésiques. La pression inspiratoire initiale était de $10 \mathrm{cmH}_{2} \mathrm{O}$ et a été augmentée par incréments de $2 \mathrm{cmH}_{2} \mathrm{O}$ 
jusqu'à ce qu'une insufflation gastrique soit détectée par échographie gastrique ou auscultation épigastrique. Le critère d'évaluation principal était la différence de pression inspiratoire entraînant une insufflation entre les deux groupes. Les méthodes diagnostiques de détection précoce de l'insufflation gastrique ont été également évaluées.

Résultats Il n’y a pas eu de différence significative concernant la valeur médiane [écart interquartile] de la pression inspiratoire induisant une insufflation gastrique entre le groupe non-NM $(n=52)$ et le groupe $N M(n=60)$ (18 [16-18] $\mathrm{cm} \mathrm{H}_{2} \mathrm{O}$ contre 18,0 [16-20] $\mathrm{cmH}_{2} \mathrm{O}$; différence des médianes, $\mathrm{O} \mathrm{cmH}_{2} \mathrm{O}$; intervalle de confiance [IC] à $95 \%: 0$ à 2; $P=0,57)$. L'incidence de l'insufflation gastrique a augmenté avec l'augmentation de la pression inspiratoire. L'insufflation gastrique a d'abord été détectée par l'échographie dans $44 \%$ des cas, et par auscultation épigastrique dans $19 \%$ des cas dans le groupe non-NM (différence en pourcentage, $25 \%$; IC à $95 \%, 6$ à 42; $P=$ 0,006) et par échographie dans $73 \%$ et par auscultation épigastrique dans $7 \%$ des cas dans le groupe NM (différence en pourcentage, $66 \%$; IC à $95 \%, 50$ à 78; $P=0,001$ ).

Conclusions Un agent bloqueur neuromusculaire n'a qu'un effet minime sur la pression inspiratoire entraînant une insufflation gastrique au cours de la ventilation au masque chez des enfants.

Enregistrement de l'essai clinique $w w w$.clinicaltrials. gov (NCT02471521); enregistré le 15 juin 2015.

Face mask ventilation is a basic procedure for oxygenation and carbon dioxide elimination when the airway is unprotected. Nevertheless, gastric insufflation during face mask ventilation can result in adverse effects, such as gastric distension, pulmonary aspiration, and ventilatory disturbance. ${ }^{1-6}$ Therefore, early detection and avoidance of gastric insufflation can prevent unfavourable events during face mask ventilation.

Gastric insufflation can be detected by either epigastric auscultation or gastric ultrasonography. The reliability of epigastric auscultation to assess air entry into the stomach was shown in a previous study performed in adults. ${ }^{7}$ Gastric ultrasonography is another useful tool for measuring gastric volume in a cross-sectional area. ${ }^{8,9}$ According to an adult study, ${ }^{10}$ gastric ultrasonography showed a higher sensitivity than epigastric auscultation for detection of gastric insufflation; however, there are no data in children.

Gastric insufflation can be induced by several factors, such as high inspiratory pressure, high inspiratory flow rate, short inspiratory time, lower oesophageal sphincter tone, high airway resistance, poor pulmonary compliance, and inadequate skill managing face mask ventilation. Among these, the inspiratory pressure is clinically easy to control during manual ventilation. Although the inspiratory pressure should be as low as possible to avoid gastric insufflation, low inspiratory pressure may yield an insufficient tidal volume. Accordingly, the optimal inspiratory pressure is the pressure that delivers adequate tidal volume without causing gastric insufflation.

The pressure causing gastric insufflation might be affected by use of a muscle relaxant. Nevertheless, there are limited data regarding the effect of a muscle relaxant on gastric insufflation during face mask ventilation. Furthermore, the optimal inspiratory pressure has not been determined in children. The purpose of this study was to evaluate the effect of a muscle relaxant on inspiratory pressure causing gastric insufflation and to determine the inspiratory pressure that minimized gastric insufflation while guaranteeing a tidal volume of 6-10 $\mathrm{mL} \cdot \mathrm{kg}^{-1}$ during face mask ventilation in children.

\section{Methods}

Patients and study protocol

Ethical approval for this study was provided by the institutional review board of Seoul National University Hospital (Seoul, Republic of Korea; numbers: H1412-083633; date of approval: 3 February 2015). This study protocol was registered at www.clinicaltrials.gov (NCT02471521). The trial was registered after recruitment began because of an error, but the study protocol was not changed.

This prospective randomized single-centre study was performed in a tertiary children's hospital in Republic of Korea from February to December 2015. Children $\leq$ five years of age who were scheduled for general anesthesia were enrolled. One day before each surgery, a staff member met with each child's parents, explained the study protocol, and then obtained written informed consent from the parents. Exclusion criteria were as follows: age < one month; body mass index $>30 \mathrm{~kg} \cdot \mathrm{m}^{-2}$; American Society of Anesthesiologists physical status $>$ II; history of intraoral or airway surgery, including tonsillectomy; history of upper airway obstruction; history of respiratory disease

Peripheral intravenous access was established in all children prior to induction of anesthesia. Patients entered the operating room without premedication. Monitoring included electrocardiography, pulse oxygen saturation $\left(\mathrm{SpO}_{2}\right)$, non-invasive blood pressure, and end-tidal carbon dioxide $\left(\mathrm{ETCO}_{2}\right)$. With the child in the supine position, anesthesia was induced using atropine $\left(0.02 \mathrm{mg} \cdot \mathrm{kg}^{-1}\right)$ and 
thiopental sodium $\left(5 \mathrm{mg} \cdot \mathrm{kg}^{-1}\right)$. Then, sevoflurane $4-5$ vol\% was administered under spontaneous ventilation. After patients showed loss of consciousness, the baseline antral area for each patient was measured using the Logiq $e$ US (GE Healthcare, Wauwatosa, WI, USA) with a 4-8 $\mathrm{MHz}$ transducer. If air in the stomach hindered capturing clear ultrasound images, a suction catheter was inserted to remove the air in the stomach.

Patients were randomly assigned to either the neuromuscular blocker (NM) or non-neuromuscular (nonNM) group. Strata were constructed based on age, and a randomization scheme was performed separately within each stratum. Block randomization was done to ensure balance of the age groups. A random allocation sequence was performed by a research assistant who prepared coded and sealed opaque envelopes for allocation concealment. The assistant then opened the envelopes after the enrolment.

After baseline gastric ultrasound measurement, the NM group received rocuronium $\left(0.6 \mathrm{mg} \cdot \mathrm{kg}^{-1}\right)$ and no neuromuscular blocking drug was given to the non-NM group. A face mask was applied tightly using a two-handed mask-hold technique with jaw thrust, with $4-8$ vol\% of sevoflurane in $100 \%$ oxygen. In the NM group, pressurecontrolled ventilation was commenced when spontaneous respiration or the single twitch response using a TOFWatch ${ }^{\circledR}-S X$ monitor (Organon Ireland Ltd., Dublin, Ireland) disappeared. In the non-NM group, pressurecontrolled ventilation was initiated after respiratory effort disappeared (measured tidal volume $<3 \mathrm{~mL} \cdot \mathrm{kg}^{-1}$ or no chest movement) to avoid unsynchronized ventilation. The initial inspiratory pressure was $10 \mathrm{cmH}_{2} \mathrm{O}$, and this was gradually increased by $2 \mathrm{cmH}_{2} \mathrm{O}$ after five consecutive breaths until gastric insufflation was detected by ultrasonography, auscultation, or both. Pressure-controlled ventilation was performed via one type of mechanical ventilator (Primus ${ }^{\circledR}$, Drager, USA) using a circle breathing system to standardize the measurement of expiratory tidal volume. The tidal volumes were recorded by a non-blinded anesthesiologist. Five consecutive tidal volumes were averaged for each inspiratory pressure. The initial respiratory rate was set at 25 breaths $\mathrm{min}^{-1}$ in infants and 20 breaths $\cdot \min ^{-1}$ in children $>$ one year of age. The inspiratory-to-expiratory ratio was 1:2 with no positive end-expiratory pressure. Blood oxygen saturation, expiratory tidal volume, and $\mathrm{ETCO}_{2}$ were continuously monitored and recorded.

A gastric antrum image was recorded in the sagittal or the parasagittal plane between the left lobe of the liver and the pancreas at the level of the aorta or inferior vena cava. The transducer was tilted and rotated perpendicular to the long axis of the antrum. After determining the longitudinal (D1) and anteroposterior (D2) diameters of the gastric antrum, the cross-sectional area (CSA) was calculated as follows, assuming that the antrum had an elliptical shape: $\mathrm{CSA}=\mathrm{D} 1 \times \mathrm{D} 2 \times \pi / 4 .{ }^{10}$

The primary outcome was the difference in the inspiratory pressure that causes gastric insufflation. We defined an adequate tidal volume as $6-10 \mathrm{~mL} \cdot \mathrm{kg}^{-1} \cdot{ }^{10}$ For assessment of gastric insufflation, a single anesthesiologist performed real-time ultrasonography of the gastric antrum, and an independent anesthesiologist performed epigastric auscultation simultaneously. Occurrence of gastric insufflation on ultrasonography was defined by the appearance of an acoustic shadow phenomenon and/or a comet-tail artefact in the antrum (Fig. 1). ${ }^{10}$ When a gurgling sound was heard during auscultation, gastric insufflation was considered to have occurred. The increase in inspiratory pressure was halted when gastric insufflation was detected by one or both methods. The experiment was ceased at an inspiratory pressure of $24 \mathrm{cmH}_{2} \mathrm{O}$ if gastric insufflation had not occurred. After all the parameters had been measured, gastric air was suctioned to decompress the stomach.

\section{Statistical analysis}

Sample size was calculated using the difference in an inspiratory pressure causing gastric insufflation between the NM and non-NM groups. An inspiratory pressure of 2 $\mathrm{cmH}_{2} \mathrm{O}$ was considered clinically significant. The expected variability in inspiratory pressure causing gastric insufflation was set as 3.5 , based on a previous study. ${ }^{2}$ The required sample size was about 60 per group, using PASS software 2008 (version 8.0.16; NCSS statistical software, Kaysville, UT, USA), with an alpha error of 0.05 , power of 0.8 , and estimated $20 \%$ attrition rate. Statistical analyses were performed using SPSS version 20 (IBM Corp., Armonk, NY, USA). The inspiratory pressure that induced gastric insufflation and the tidal volumes at each inspiratory pressure were investigated using descriptive analysis.

The differences in continuous variables between the NM group and non-NM group were evaluated using Student's $t$ test or the Mann-Whitney $U$ test. Patients were further subdivided into the following three subgroups according to age: 1) from $>$ one month to six months, 2) from $>$ six months to one year, and 3) from $>$ one year old to five years of age. In each subgroup, differences among the subgroups were analyzed using analysis of variance or a Kruskal-Wallis test with a Bonferroni correction. Comparison of categorical data among the subgroups was performed with a $\chi^{2}$ or Fisher's exact test. The percentage difference with $95 \%$ confidence interval (CI) was calculated according to previous reports. ${ }^{11,12} \mathrm{~A}$ threshold of $P<0.05$ was set to indicate statistical significance. 
A

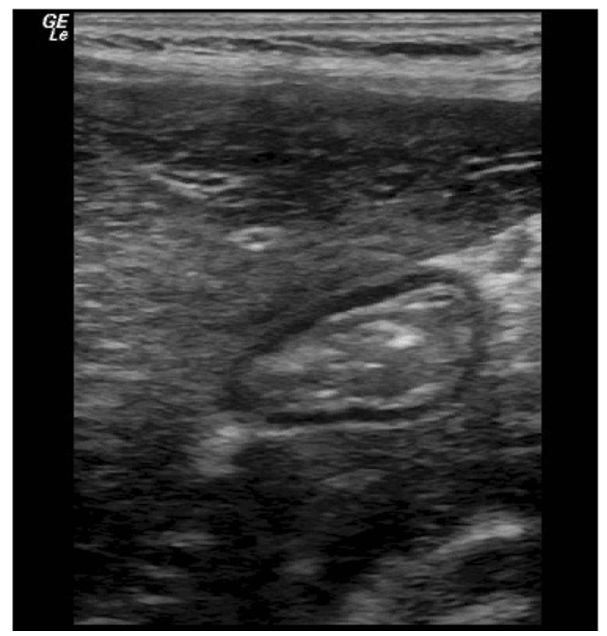

Fig. 1 Ultrasound image of the gastric antrum in the epigastric area obtained in a sagittal or parasagittal plane. A) A cross-sectional view of the antrum before face mask ventilation. B) An acoustic shadow

\section{Results}

A total of 120 children participated in the present study. In eight patients in the non-NM group, it was not possible to visualize the gastric antrum because of pre-existence of gastric air, and we could not detect additional air entrapment during face mask ventilation. Therefore, we excluded these eight patients from data analysis, and 112 patients (52 children in the non-NM group and 60 children in the NM group) were analyzed. There were no additional missing data (Fig. 2).

The characteristics of patients and the variables associated with gastric insufflation are presented in Table 1. There were no significant differences in the median (interquartile range [IQR]) inspiratory pressure inducing gastric insufflation between the non-NM and the $\mathrm{NM}$ groups (18 [16-18] $\mathrm{cmH}_{2} \mathrm{O}$ vs 18 [16-20] $\mathrm{cmH}_{2} \mathrm{O}$; median difference, $0 \mathrm{cmH}_{2} \mathrm{O}$; $95 \% \mathrm{CI}, 0$ to $2, P=0.57$ ). Ultrasonography detected gastric insufflation more sensitively than auscultation. In the non-NM group, gastric insufflation was first detected by ultrasonography in $44 \%$ of cases and by epigastric auscultation in $19 \%$ of cases (percentage difference, 25\%;95\% CI, 6 to $42 ; P=$ 0.006). In the NM group, gastric insufflation was first detected by ultrasonography in $73 \%$ of cases and by epigastric auscultation in $7 \%$ of cases (percentage difference, 66\%; 95\% CI, 50 to 78; $P<0.001$ ). All cases of gastric insufflation that occurred at an inspiratory pressure $\leq 14 \quad \mathrm{cmH}_{2} \mathrm{O}$ were first detected by ultrasonography. After one or two more ventilations, gastric insufflation of all patients could be detected by

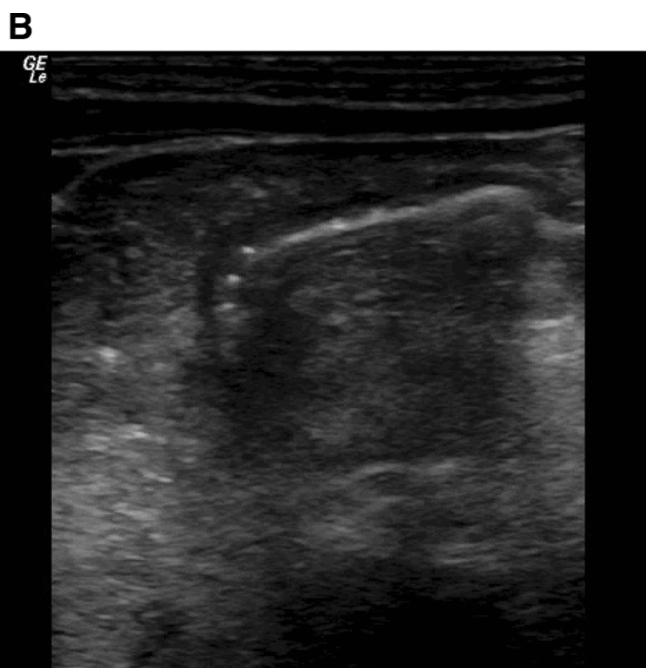

phenomenon and/or a comet-tail artefact into the antrum, representing entry of air into the stomach

both methods. The median baseline CSA of the gastric antrum was similar between the non-NM group and the $\mathrm{NM}$ group ( 0.8 and $0.7 \mathrm{~cm}^{2}$, respectively), and both of these values were increased after gastric insufflation (1.2 $\mathrm{cm}^{2}$ for both groups).

Table 2 shows the results of the subgroup analysis. There were no significant differences in the inspiratory pressure that induced gastric insufflation among the different age groups.

Figure 3 shows the incidence of gastric insufflation and the tidal volume at each inspiratory pressure. The incidence of gastric insufflation increased with increasing inspiratory pressure. At an inspiratory pressure of $16 \mathrm{cmH}_{2} \mathrm{O}$, the incidence of gastric insufflation was 37\% (95\% CI, 27 to $47 \%$ ) in the non-NM group and 33\% (95\% CI, 24 to 44\%) in the NM group. An adequate tidal volume (tidal volume 6-10 $\mathrm{mL} \cdot \mathrm{kg}^{-1}$ ) with an absence of gastric insufflation was observed in 56\% (99\% CI, 43 to $68 \%$ ) of the participants at an inspiratory pressure of $10 \mathrm{cmH}_{2} \mathrm{O}$, in $71 \%$ (99\% CI, 58 to $81 \%$ ) at an inspiratory pressure of $12 \mathrm{cmH}_{2} \mathrm{O}$, in $71 \%$ $(99 \% \mathrm{CI}, 58$ to $81 \%$ ) at an inspiratory pressure of 14 $\mathrm{cmH}_{2} \mathrm{O}$, in $55 \%(99 \% \mathrm{CI}, 42$ to $67 \%)$ at an inspiratory pressure of $16 \mathrm{cmH}_{2} \mathrm{O}$, in $33 \%$ (99\% CI, 22 to $46 \%$ ) at an inspiratory pressure of $18 \mathrm{cmH}_{2} \mathrm{O}$, and in no patients at an inspiratory pressure of $20 \mathrm{cmH}_{2} \mathrm{O}$.

Two patients in the NM group showed a minimum pulse oximeter value of $96 \%$ at inspiratory pressures of 10 $\mathrm{cmH}_{2} \mathrm{O}$ and $12 \mathrm{cmH}_{2} \mathrm{O}$. No other complications, including laryngospasm, bronchospasm, or desaturation $<96 \%$, were observed during the experimental period or during patient recovery. 


\section{Enrollment}

Assessed for eligibility $(n=120)$

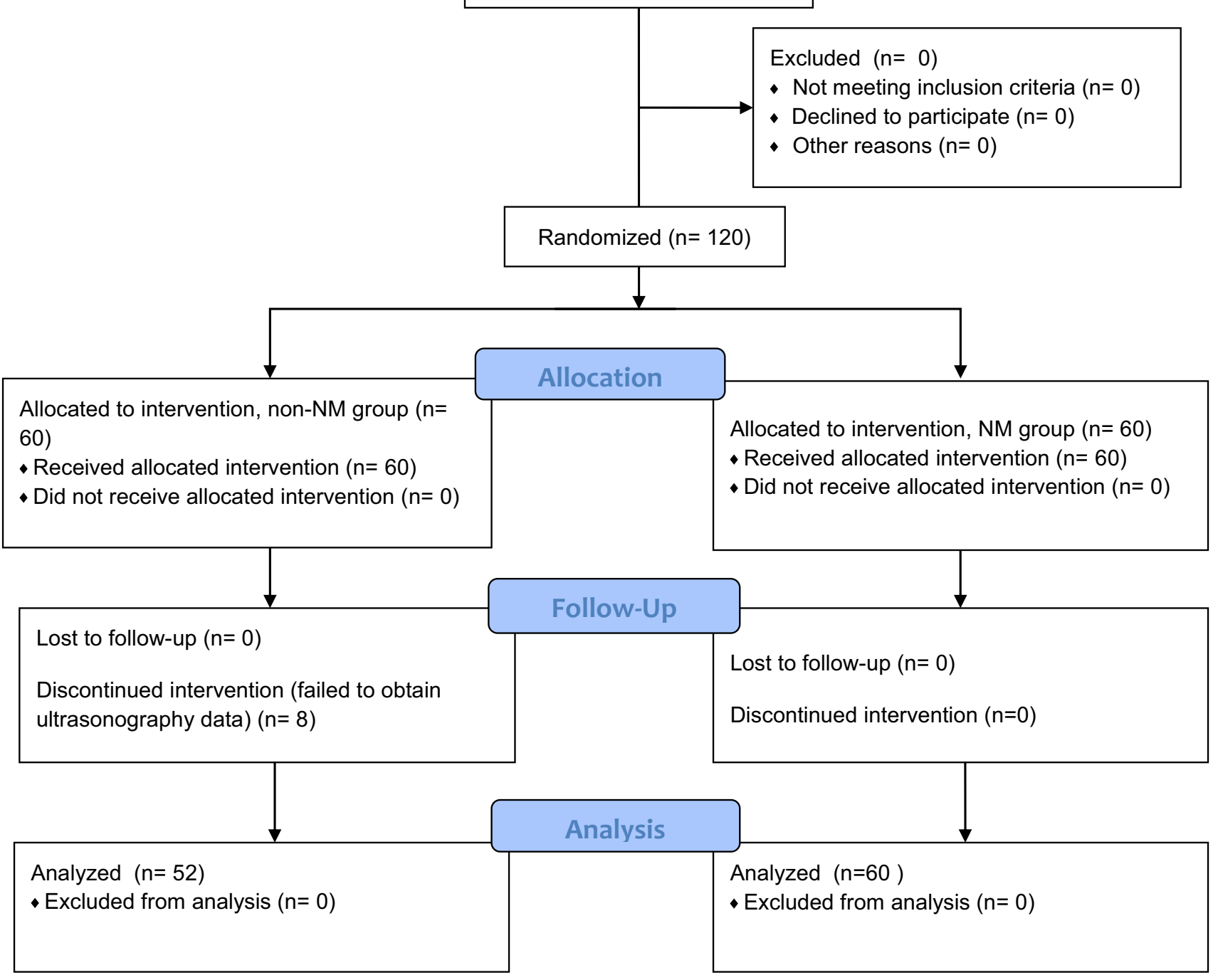

Fig. 2 Consolidated standards of reporting trials (CONSORT) diagram. Non-NM group = non-neuromuscular blocker group; NM group = neuromuscular blocker group

\section{Discussion}

In this study of children who underwent general anesthesia, we found that the inspiratory pressure that induced gastric insufflation during face mask ventilation was similar, regardless of whether a neuromuscular blocker was used. An adequate tidal volume with an absence of gastric insufflation was most often observed at inspiratory pressure of 12 and $14 \mathrm{cmH}_{2} \mathrm{O}$. Our results were similar to those of a recent pediatric study, which showed that an inspiratory pressure of $12 \mathrm{cmH}_{2} \mathrm{O}$ was optimal to provide adequate ventilation with a lower incidence of gastric insufflation in paralyzed children. ${ }^{13}$

Our study showed that gastric insufflation occurs at pressures $<20 \mathrm{cmH}_{2} \mathrm{O}$ in most children. When comparing the results of the present study with adult data, the incidence of gastric insufflation was higher in children than in adults at the same levels of inspiratory pressure $(96 \% \mathrm{vs}$ $53 \%$ at an inspiratory pressure of $\left.20 \mathrm{cmH}_{2} \mathrm{O}\right) .{ }^{10}$ This difference may be due to the shorter length of the esophagus and smaller airway size in children. Therefore, special attention to the inspiratory pressure during face mask ventilation in children is required, as the risk of gastric insufflation at the same inspiratory pressure is greater than in adults.

We found no significant difference in airway pressure leading to gastric insufflation between the NM and the nonNM groups. Several explanations may account for this similarity observed between the NM and the non-NM groups. First, both inhalational ${ }^{14}$ and intravenous 
Table 1 Characteristics of patients and variables associated with gastric insufflation

\begin{tabular}{|c|c|c|c|c|}
\hline Variables & $\begin{array}{l}\text { Non-NM group }(n= \\
52)\end{array}$ & $\begin{array}{l}\text { NM group }(n= \\
60)\end{array}$ & $\begin{array}{l}\text { Mean difference } \\
(95 \% \mathrm{CI})\end{array}$ & $\begin{array}{l}P \\
\text { value }\end{array}$ \\
\hline Age $(y r)$, mean $(S D)$ & $1.4(1.2)$ & $1.2(1.1)$ & $0.1(-0.3$ to 0.6$)$ & 0.49 \\
\hline Height $(\mathrm{cm})$, mean $(\mathrm{SD})$ & $79(14)$ & $76(14)$ & $3(-2$ to 8$)$ & 0.29 \\
\hline Weight (kg), mean (SD) & $10.4(3.5)$ & $10.0(3.8)$ & $0.4(-1.0$ to 1.7$)$ & 0.60 \\
\hline Sex & & & & 0.34 \\
\hline Male, $n(\%)$ & $31(60)$ & $39(65)$ & & \\
\hline Female, $n(\%)$ & $21(40)$ & $21(35)$ & & \\
\hline Inspiratory pressure inducing gastric insufflation $\left(\mathrm{cmH}_{2} \mathrm{O}\right)$, median [IQR] & $18[16-18]$ & $18[16-20]$ & $0[0-2]^{*}$ & 0.57 \\
\hline $\begin{array}{l}\text { Minimal inspiratory pressure for tidal volume } 6-10 \mathrm{~mL} \cdot \mathrm{kg}^{-1}\left(\mathrm{cmH}_{2} \mathrm{O}\right) \text {, } \\
\text { median [IQR] }\end{array}$ & $10[7-12]$ & $11[8-12]$ & $-0.1[-0.3 \text { to }-0.1]^{*}$ & 0.06 \\
\hline First detection of gastric insufflation & & & & 0.03 \\
\hline Ultrasound, $n(\%)$ & $23(44)$ & $44(73)$ & $29(11$ to 50$) \dagger$ & 0.002 \\
\hline Auscultation, $n(\%)$ & $10(19)$ & $4(7)$ & $12(-26$ to 0$) \dagger$ & 0.06 \\
\hline Simultaneous detection by both methods, $n(\%)$ & $19(37)$ & $12(20)$ & $17(-1$ to 23$) \dagger$ & 0.05 \\
\hline
\end{tabular}

*Median difference $(95 \% \mathrm{CI})$. $\dagger$ Percentage difference $(95 \% \mathrm{CI})$

$\mathrm{CI}=$ confidence interval; $\mathrm{IQR}=$ interquartile range; $\mathrm{NM}$ group = neuromuscular blocker group; non-NM group = non-neuromuscular blocker group; $\mathrm{SD}=$ standard deviation

Table 2 Inspiratory pressure inducing gastric insufflation $\left(\mathrm{cmH}_{2} \mathrm{O}\right)$ according to age group

\begin{tabular}{|c|c|c|c|c|}
\hline & Non-NM group $(n=52)$ & NM group $(n=60)$ & Mean difference $(95 \% \mathrm{CI})$ & $P$ value \\
\hline \multicolumn{5}{|l|}{ Age subgroup } \\
\hline 1 month $<$ age $\leq 6$ months $(n=36)$ & $18[16-20]$ & $18[18-20]$ & $0(-1$ to 1$)$ & 0.64 \\
\hline 6 months $<$ age $\leq 1 \mathrm{yr}(n=38)$ & $18[18-20]$ & $18[16-20]$ & $1(-1$ to 2$)$ & 0.57 \\
\hline $1 \mathrm{yr}<$ age $\leq 5 \mathrm{yr}(n=38)$ & $18[16-18]$ & $18[14-20]$ & $-1(-3$ to 2$)$ & 0.50 \\
\hline
\end{tabular}

Data are presented as median [interquartile ranges]

In the non-NM group, the number of patients aged between 1 and 6 months, 6 months and 1 year, and 1 year and 5 years was 16,18 , and 18 respectively

$\mathrm{CI}=$ confidence interval; $\mathrm{NM}$ group = neuromuscular blocker group; non-NM group = non-neuromuscular blocker group

$P$ value is for the difference between non-NM and NM groups

anesthetics $^{15}$ can significantly reduce the mean sphincter tone even without the use of a neuromuscular blocker. Anesthesia itself may therefore increase the risk of gastric insufflation during face mask ventilation because of this decrease in the upper esophageal sphincter tone. ${ }^{15,16}$ In addition, previous studies showed no significant decrease in esophageal pressure after administration of a neuromuscular blocker. ${ }^{17,18}$ Ahlstrand et al. found that the barrier pressure, the difference between the pressure in the lower esophageal sphincter and intragastric pressure, measured by manometry decreased after anesthetic induction using propofol, fentanyl, and sevoflurane, but following muscle relaxation with rocuronium showed no significant changes in barrier pressure. ${ }^{18}$ Finally, a twohand mask and jaw thrust technique with the oral airway could cause the conditions for face mask ventilation to be similar between paralyzed and non-paralyzed children.

Along with this insignificant influence of NM, we also observed no significant differences in the inspiratory pressure that induced gastric insufflation among the different age groups. This result differs from that of a previous study, which reported that a lower inspiratory pressure induces gastric insufflation in younger children. ${ }^{19}$ Nevertheless, this study evaluated gastric insufflation only by epigastric auscultation, while our study used two separate methods. The detection rate of gastric insufflation was higher when both ultrasonography and auscultation were used than when auscultation alone was used. For example, a previous study reported that gastric insufflation was detected by auscultation in only $58 \%$ of 

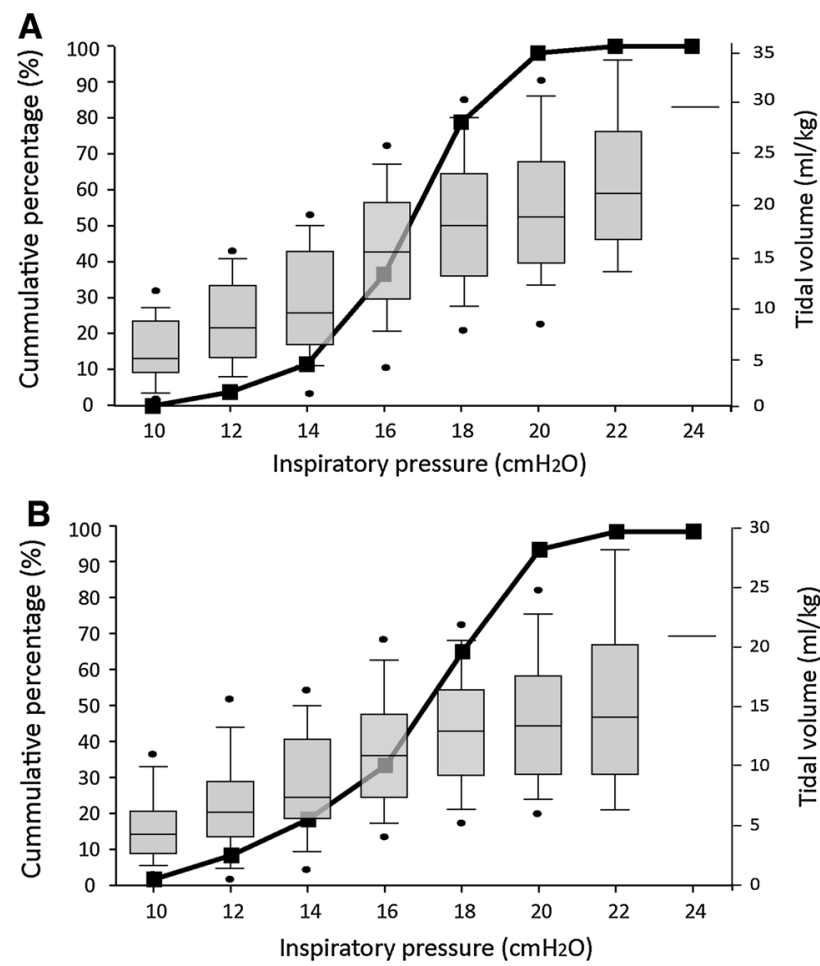

Fig. 3 The incidence of gastric insufflation and the median tidal volumes at each inspiratory pressure A) Non-neuromuscular blocker group; B) neuromuscular blocker group. The black line with the inner square represents the incidence of gastric insufflation, which increases with increased inspiratory pressure. The box and whisker plots represent the tidal volumes at each inspiratory pressure. Lines within the boxes represent the median value of the tidal volumes

children at an inspiratory pressure of $20 \mathrm{cmH}_{2} \mathrm{O},{ }^{19}$ similar to the 53\% detection rate reported by both ultrasound and auscultation in an adult study. ${ }^{10}$ Nevertheless, the detection rate of gastric insufflation in children was increased to $96 \%$ using two methods in the present study. Another reason for the negligible effect of age could be explained by the finding of a previous study that reported that, although the length of the lower esophageal sphincter increases with age, lower esophageal sphincter tone is well developed by two weeks of age, and infants without gastroesophageal reflux show greater lower esophageal sphincter pressure (mean $43.3 \mathrm{mmHg}$ ) than children older than one year of age without gastroesophageal reflux (mean $30.6 \mathrm{mmHg}$ ) ${ }^{20}$

We found that gastric ultrasonography was more sensitive than epigastric auscultation for detecting gastric insufflation in children. Our results were similar to those of previous studies that reported that $18-50 \%$ of cases of gastric insufflation detected by ultrasonography were missed when using epigastric auscultation. ${ }^{10,21}$ This is because, compared with gastric ultrasonography, a larger volume of air entering the stomach was required for the gurgling sound to be heard during auscultation. ${ }^{7,21}$ On the other hand, the rate of first detection of gastric insufflation using ultrasound was higher in the NM group than in the non-NM group. This could be explained by the effect of the neuromuscular blocker. If a small amount of air entered into the stomach, subtle movement or respiratory effort of the patients may hinder the detection of gastric insufflation using ultrasonography in non-NM groups. Therefore, we assumed that gastric insufflation could be detected more sensitively using ultrasonography in paralyzed patients.

There were some limitations to the present study. First, we did not assess the sensitivity and specificity of gastric ultrasonography for the detection of stomach air. In addition, we did not perform a quantitative analysis to detect a minimal amount of air. Second, although we tried to remove air from the stomach, it was difficult to obtain the baseline image of the gastric antrum using ultrasonography, because some air remained in the stomach in some patients. This was more common in the non-NM group because of the longer duration of selfbreathing than in the NM group. Third, anesthesiologists who performed the mask ventilation and outcome assessor could recognize the group allocation because of subtle movement, respiratory effort, or a longer duration of selfbreathing; accordingly, blinding was not perfect. Fourth, we used thiopental sodium in all patients instead of propofol; the differences among these agents in terms of the influence on airway response should be considered before generalization of our results. Lastly, the sample size calculation for subgroup analysis for inspiratory pressure inducing gastric insufflation according to age was not performed.

\section{Conclusions}

In summary, we found that use of a neuromuscular blocker had little effect on the inspiratory pressure level associated with gastric insufflation. An inspiratory pressure of 12-14 $\mathrm{cmH}_{2} \mathrm{O}$ minimized the occurrence of gastric insufflation while delivering an adequate tidal volume during face mask ventilation in pediatric patients. Face mask ventilation should be carefully performed in children, regardless of the use of a neuromuscular blocker, because gastric insufflation can easily occur in children.

Conflict of interest None declared.

Editorial responsibility This submission was handled by Dr. Gregory L. Bryson, Deputy Editor-in-Chief, Canadian Journal of Anesthesia.

Author Contributions Jin-Tae Kim and Ji-Hyun Lee contributed substantially to all aspects of this manuscript, including conception and design; acquisition, analysis, and interpretation of data; and drafting the article. Eun-Hee Kim contributed substantially to the conception and design of the manuscript. In-Kyung Song contributed 
substantially to the acquisition of data. Hee-Soo Kim contributed to the analysis of data. Haesun Jung contributed substantially to the interpretation of data.

Funding Support was provided solely from institutional and/or department sources.

\section{References}

1. Weiler $N$, Heinrichs $W$, Dick W. Assessment of pulmonary mechanics and gastric inflation pressure during mask ventilation. Prehosp Disaster Med 1995; 10: 101-5.

2. Weiler $N$, Latorre $F$, Eberle B, Goedecke R, Heinrichs W. Respiratory mechanics, gastric insufflation pressure, and air leakage of the laryngeal mask airway. Anesth Analg 1997; 84: 1025-8.

3. Ho-Tai LM, Devitt JH, Noel AG, O'Donnell MP. Gas leak and gastric insufflation during controlled ventilation: face mask versus laryngeal mask airway. Can J Anaesth 1998; 45: 206-11.

4. Neelakanta $G$, Chikyarappa A. A review of patients with pulmonary aspiration of gastric contents during anesthesia reported to the Departmental Quality Assurance Committee. J Clin Anesth 2006; 18: 102-7.

5. Seet MM, Soliman KM, Sbeih ZF. Comparison of three modes of positive pressure mask ventilation during induction of anaesthesia: a prospective, randomized, crossover study. Eur J Anaesthesiol 2009; 26: 913-6.

6. Paal P, Neurauter A, Loedl M, et al. Effects of stomach inflation on haemodynamic and pulmonary function during cardiopulmonary resuscitation in pigs. Resuscitation 2009; 80: 365-71.

7. Brimacomb J, Keller C, Kurian S, Myles J. Reliability of epigastric auscultation to detect gastric insufflation. Br J Anaesth 2002; 88: 127-9.

8. Perlas A, Chan VW, Lupu CM, Mitsakakis N, Hanbidge A. Ultrasound assessment of gastric content and volume. Anesthesiology 2009; 111: 82-9.

9. Perlas A, Davis L, Khan M, Mitsakakis N, Chan VW. Gastric sonography in the fasted surgical patient: a prospective descriptive study. Anesth Analg 2011; 113: 93-7.
10. Bouvet L, Albert ML, Augris C, et al. Real-time detection of gastric insufflation related to facemask pressure-controlled ventilation using ultrasonography of the antrum and epigastric auscultation in nonparalyzed patients: a prospective, randomized, double-blind study. Anesthesiology 2014; 120: 326-34.

11. Wilson EB. Probable inference, the law of succession, and statistical inference. J Am Stat Assoc 1927; 22: 209-12.

12. Newcombe $R G$. Interval estimation for the difference between independent proportions: comparison of eleven methods. Stat Med 1998; 17: 873-90.

13. Qian $X, H u Q$, Zhao $H$, et al. Determination of the optimal inspiratory pressure providing adequate ventilation while minimizing gastric insufflation using real-time ultrasonography in Chinese children: a prospective, randomized, double-blind study. BMC Anesthesiol 2017; 17: 126.

14. Thorn K, Thorn SE, Wattwil M. The effects on the lower esophageal sphincter of sevoflurane induction and increased intra-abdominal pressure during laparoscopy. Acta Anaesthesiol Scand 2006; 50: 978-81.

15. Vanner $R G$, Pryle BJ, O'Dwyer JP, Reynolds F. Upper oesophageal sphincter pressure and the intravenous induction of anaesthesia. Anaesthesia 1992; 47: 371-5.

16. McGrath JP, McCaul C, Byrne PJ, Walsh TN, Hennessy TP. Upper oesophageal sphincter function during general anaesthesia. Br J Surg 1996; 83: 1276-8.

17. Hunt PC, Cotton BR, Smith G. Barrier pressure and muscle relaxants. Comparison of the effects of pancuronium and vecuronium on the lower oesophageal sphincter. Anaesthesia 1984; 39: 412-5

18. Ahlstrand R, Thorn SE, Wattwil M. High-resolution solid-state manometry of the effect of rocuronium on barrierpressure. Acta Anaesthesiol Scand 2011; 55: 1098-105.

19. Lagarde $S$, Semjen F, Nouette-Gaulain $K$, et al. Facemask pressure-controlled ventilation in children: what is the pressure limit? Anesth Analg 2010; 110: 1676-9.

20. Moroz SP, Espinoza J, Cumming WA, Diamant NE. Lower esophageal sphincter function in children with and without gastroesophageal reflux. Gastroenterology 1976; 71: 236-41.

21. Park JH, Kim JY, Lee JM, Kim YH, Jeong HW, Kil HK. Manual vs. pressure-controlled facemask ventilation for anaesthetic induction in paralysed children: a randomised controlled trial. Acta Anaesthesiol Scand 2016; 60: 1075-83. 